\title{
Escritores hispanos en la Roma de Augusto
}

\author{
Antonio Alvar EzQuerra \\ Universidad de Alcalá \\ antonio.alvar@uah.es
}

\section{RESUMEN}

Revisión de la relación entre las provincias hispanas y Roma por lo que respecta a la actividad intelectual y, en particular, a la de los oradores y escritores de origen hispano en la época de Augusto, desde el final de la guerra civil entre César y Pompeyo a la muerte del emperador. Se presta especial atención a los Balbos, a Séneca el rétor, a los escritores y oradores hispanos mencionados por él en su obra y, finalmente, a Higino.

Palabras clave: Lucio Cornelio Balbo el Viejo. Lucio Cornelio Balbo el Joven. Lucio Anneo Séneca el Viejo (o el rétor). Grammatici. Escritores y oradores hispanos. Gayo Julio Higino.

\section{Hispanic Authors in the Rome of Augustus}

\begin{abstract}
Update of the relationship between the provinces of Hispania and Rome regarding the intellectual activity and, in particular, the writers and orators of Hispanic origin during the Augustean period, from the end of the civil war between Caesar and Pompey and the death of the emperor. The Balbi, Seneca the Elder, the writers and orators mentioned by him in his works, and Higinus will receive special attention.
\end{abstract}

Key Words: Lucius Cornelius Balbus Sr. Lucius Cornelius Balbus Jr. Lucius Annaeus Seneca Sr. (other, the rhetor). Grammatici. Writers and public speakers from Hispania. Gaius Julius Higinus. 
Es bien sabido ${ }^{1}$ que, desde que Roma extendió sus intereses fuera de la Península Itálica, todos aquellos miembros de las élites provinciales de origen no romano que quisieran aspirar a jugar algún papel de importancia en sus respectivas comunidades de origen, debían romanizarse - es decir, debían asimilarse a las formas de vida impuestas por el poder de Roma- y debían latinizarse - es decir, debían poseer un alto nivel de conocimiento de la lengua latina-; tal proceso de romanización y de latinización era progresivo y raramente se lograba con plenitud en el plazo de una sola generación. Obviamente, tal proceso de romanización y de latinización era más rápido y exitoso si se cumplía, en todo o en parte, en la propia Roma. De modo que resulta habitual observar cómo las élites provinciales envían a sus jóvenes miembros a la capital para educarse e instruirse de manera profunda en el nuevo sistema de valores y en el nuevo marco de relaciones de poder; naturalmente, esa romanización conllevaba una mejor promoción de los jóvenes en la vida pública provincial y, en ocasiones, también en la del propio Estado romano. Del mismo modo que en los decenios precedentes los jóvenes de las familias ilustres de Roma completaron su formación en tierras griegas, ahora son los jóvenes provinciales los que van a Roma a completar su adiestramiento para la vida pública -la milicia, el foro, los negocios-. Y también es bien sabido que esa formación incluía de manera preeminente la formación retórica, en la que se exigía no solo una alta capacidad dialéctica sustentada en una profunda formación intelectual, sino también una exquisita dicción y un uso apropiado del lenguaje. En caso contrario, el origen provincial podría pesar de manera irreparable, limitando las expectativas de promoción. A este respecto se suele recordar cómo Cicerón se burla de Quinto Cecilio ${ }^{2}$ que pretende ser tenido por alguien respetable en la Ciudad y discutir en un tribunal romano, siendo así que había aprendido su griego y su latín en Sicilia; o cómo el propio Cicerón caracterizaba a los poetas cordobeses como pingue quiddam sonantes atque peregrinum; ${ }^{3}$ o cómo el purista Mesala Corvino decía de manera irónica a propósito de Porcio Latrón, el gran rétor de origen hispano, amigo de Séneca el rétor, que era elocuente sua lingua, por más que no podamos deducir de tan ácido comentario qué tipo de errores -de pronunciación, gramaticales, léxicos- cometía el maestro; ${ }^{4}$ o también, cómo el propio Séneca el rétor reprochaba a su

1 Vid. v. gr. GuALANDRI 1989, en especial 476-484 (“2. L'età repubblicana e il primo secolo dopo Cristo: l'attrazione di Roma").

2 Vid. Cic., Div. in Caec. 39: Dicenda, demonstranda, explicanda sunt omnia, causa non solum exponenda, sed etiam graviter copioseque agenda est; perficiendum est, si quid agere aut proficere vis, ut homines te non solum audiant, verum etiam libenter studioseque audiant. In quo si te multum natura adiuvaret, si optimis a pueritia disciplinis atque artibus studuisses et in his elaborasses, si litteras Graecas Athenis non Lilybaei, Latinas Romae non in Sicilia didicisses, tamen esset magnum tantam causam, tam exspectatam, et diligentia consequi et memoria complecti et oratione expromere et voce ac viribus sustinere.

3 Vid. Cic., Pro Arch. 26: Quid? a Q. Metello Pio, familiarissimo suo, qui civitate multos donavit, neque per se neque per Lucullos impetravisset? qui praesertim usque eo de suis rebus scribi cuperet ut etiam Cordubae natis poetis pingue quiddam sonantibus atque peregrinum tamen auris suas dederet. Nada más sabemos de esos poetas cordobeses a que se refiere Cicerón. Para las particularidades del latín de Hispania, vid. Alvar 1983, 1998 y ADAms 2007, en especial 370 y ss.

4 Vid. Sen., Contr. 2.4.8: Fuit autem Messala exactissimi ingenii quidem in omni studiorum parte, sed Latini utique sermonis observator diligentissimus; itaque cum audisset Latronem declamantem, dixit: sua lingua disertus est. Ingenium illi concessit, sermonem obiecit. Poca gracia le hizo a Porcio Latrón el comentario, de 
compatriota Sextilio Ena su manera de recitar, a propósito de la que se podría decir lo mismo que había dicho Cicerón de los poetas cordobeses de su tiempo. ${ }^{5}$

Era preciso acudir a Roma, si se pretendía hacer carrera, incluso en provincias. La nómina de personajes ilustres -políticos, escritores, hombres de negocios, etc.- que siguieron ese modelo de comportamiento es muy considerable, hasta el punto de que es ese el perfil habitual de las biografías de los notables tardorrepublicanos y de los primeros años del Imperio. Verona, Arpino, Mantua, Venusa, Padua, Sulmona enviaron -desde tierras itálicas- a sus Catulos, Cicerones, Virgilios, Horacios, Livios u Ovidios a la Ciudad Eterna, al tiempo que ya llegaban desde Fréjus o Cádiz Galos o Balbos. Pues de la Narbonense y de la Bética vinieron los primeros personajes extraitálicos que contaron en la historia de Roma. Obviamente, ese recorrido de ida, motivado por el gran desarrollo socioeconómico de las provincias de origen, facilitó que, en el de vuelta, esas mismas provincias mejoraran su desarrollo de la mano de sus élites, ya profundamente romanizadas. De modo que el proceso de romanización en Roma de las élites provinciales produjo como resultado la aceleración de los procesos de romanización provincial. Roma se enriquecía admitiendo incluso en el Senado a personajes oriundos de provincias, al tiempo que enriquecía a esos mismos personajes y, con frecuencia, a sus provincias. ${ }^{6}$ Séneca el trágico sintetiza, a otros efectos, este viaje de ida y vuelta al tratar de consolar a su madre, con motivo de su exilio, pues no es esto más que un cambio de domicilio, como el que han tenido que afrontar muchos colonos, dejando Italia para reubicarse en los territorios provinciales, al tiempo que otros muchos habitantes de las provincias -ciudadanos o no-, han tenido que dirigirse a Roma para cumplir sus ambiciones, fueran cuales fueran, o simplemente para recibir una educación adecuada. ${ }^{7}$

Por lo que respecta a Hispania, los ejemplos de las familias de los Balbos de Cádiz ${ }^{8}$ y de los Anneos de Córdoba son, por diferentes, muy sintomáticos y esclarecedores de cómo se pudieron desarrollar algunos aspectos de los procesos de romanización provincial. Los Balbos hundían sus orígenes familiares en la propia Hispania; tal vez eran púnicos vinculados de alguna manera al templo de Melkart en Gades. Los Anneos, por contra, procedían de territorio etrusco o de territorio ilirio, según denuncia su nombre, ${ }^{9}$ y se habrían afincado en tierras hispanas en algún momento de la conquista y colonización de ese territorio. Pero ni unos ni otros habrían podido, sin

modo que pro Pythodoro Messalae orationem disertissimam recitavit, [que] conpositamque suasoriam $<$ de $>$ Theodoto declamavit per triduum (ibidem). Pero ya nadie pudo quitarle el sambenito.

5 Vid. Sen., Suas. 6.27: Sextilius Ena fuit homo ingeniosus magis quam eruditus, inaequalis poeta et plane quibusdam locis talis quales esse Cicero Cordubenses poetas ait, < pingue > quiddam sonantis atque peregrinum. Cfr. Castillo 2006, 202.

6 Para el caso de Hispania, vid. por ejemplo, Castillo 1965, 1984, 1990, Caballos (passim), Weinrib 1990, Padilla Monge 2006, Saquete Chamizo 2006.

7 Sen., Dial. XII 6.4: Ex municipiis et coloniis suis, ex toto denique orbe terrarum confluxerunt: alios adduxit ambitio, alios necessitas officii publici, alios inposita legatio, alios luxuria opportunum et opulentum vitiis locum quaerens, alios liberalium studiorum cupiditas, alios spectacula; quosdam traxit amicitia, quosdam industria laxam ostendendae virtuti nancta materiam; quidam venalem formam attulerunt, quidam venalem eloquentiam.

8 Rubio 1949 y 1950, Boscs-Plateaux 1994, Rodríguez Neila 1996.

9 Cfr. GrifFin 1972. 
duda, escalar las posiciones que lograron si no hubieran desarrollado buena parte de su actividad en la propia Roma.

Lucio Cornelio Balbo el Viejo, nacido en Cádiz hacia el 95 a.C. en una noble familia indígena de origen púnico, participó desde el 79 a.C. y hasta el 72 a.C. a las órdenes de Q. Metelo Pío, primero, de Memmio después y de Pompeyo, finalmente, en las guerras contra Sertorio, lo que le valió el derecho de ciudadanía y el favor del gran general. Además, en esas campañas conoció a M. Terencio Varrón, el sabio filólogo y anticuarista romano, que había sustituido a Memmio, caído en combate, al frente de las tropas; luego, en Roma, trabó amistad con Julio César -a quien ya conocía desde su época de cuestor en Hispania, en el 68 a.C.-, a quien ayudó de manera decisiva como praefectus fabrum en su campaña hispana, que le valió la celebración de un triunfo en Roma, y fue determinante en la constitución del llamado Primer Triunvirato; facilitó la pretura de César en Hispania (61 a.C.) y fue luego, de nuevo, su praefectus fabrum en las campañas contra los galos (58 a.C.); por fin, durante la guerra civil supo sortear las dificultades de quien se encuentra entre los dos bandos pero no pudo lograr el entendimiento deseado entre César y Pompeyo; al acabar la contienda, se convirtió en el hombre fuerte del dictador, de quien ya administraba sus cuantiosos bienes, y logró la ciudadanía romana para los gaditanos (vid. la contundente afirmación de Tácito en Ann. XII 60: C. Oppius et Cornelius Balbus primi Caesaris opibus potuere condiciones pacis et arbitria belli tractare); por fin, tras el asesinato de César, defendió la causa de Octavio, que le premió nombrándole cónsul (suffectus) en el 40 a.C., convirtiéndose en el primer cónsul provincial y, además, no romano de nacimiento. Naturalmente, el difícil equilibrio que supo mantener Cornelio Balbo entre las facciones enfrentadas no le privó de tener serios enemigos que pusieron en duda sus actuaciones (vid. Plin., VII 136) y la legitimidad de su ciudadanía, por lo que tuvo que ser defendido en una causa memorable (Pro L. Cornelio Balbo oratio) por el mismísimo Cicerón en el 56 a.C. Para el orador, entre los varios Balbos conocidos en su tiempo, el gaditano era el Balbo por antonomasia, según se desprende de su correspondencia en donde es numerosas veces nombrado por el cognomen tan solo; hasta tal punto desempeñó un papel protagonista en los momentos finales de la República.

Esta estrecha relación con los hombres más poderosos de Roma, a quienes ayudó en momentos decisivos, permitió que Balbo y su familia, ya poseedores de un importante patrimonio obtenido a través del comercio, lo incrementaran entre otros procedimientos mediante la concesión y explotación de minas en el entorno de Aliseda (provincia de Cáceres); ${ }^{10}$ también se supone que los Balbos poseían una importante flota con la que controlaban, sin duda, el comercio marítimo de la fachada atlántica peninsular.

Pues bien, L. Cornelio Balbo, además de ser un muy destacado hombre de acción, con capacidades empresariales, militares y políticas, sintió un vivo interés por la filosofía y mantuvo una abundante correspondencia con César, que aún llegó a conocer

10 Es difícil precisar si una inscripción encontrada en Cáceres (AE 1962, 71. [L(ucio)] CORNELIO/ BALBO IMP(eratori)/C(olonia) - NORB(a) CAESA(rina)/ PATRONO) se refiere a él o a su sobrino, del que luego se dirá. 
Aulo Gelio; esa correspondencia, con la que se podían componer varios libros, estaba cifrada y el gramático Probo escribió un tratado con la pretensión de decodificarla; también se cambió cartas con Cicerón, algunas de las cuales se han conservado entre la correspondencia del arpinate (vid. por ejemplo, ad Att. VIII 15a; IX 7a, 7b, 13a, además de numerosas referencias a otras cartas suyas). Pero también se le recuerda como hombre de letras gracias a la redacción de un tratado histórico titulado Ephemerides, en el que se detallaban las actuaciones diarias de Julio César; esta obra, que debió ser compuesta y publicada probablemente poco después de la muerte de César, ${ }^{11}$ por desgracia, no ha llegado a nosotros. Suetonio conoció y utilizó esa obra pues cita su autoridad a propósito de los presagios que anunciaron el magnicidio de los idus de marzo (Caes. 81.2: cuius rei, ne quis fabulosam aut commenticiam putet, auctor est Cornelius Balbus, familiarissimus Caesaris) y para Sidonio Apolinar (Epist. IX 14.7: nam si omittantur quae de titulis dictatoris invicti scripta Patavinis sunt voluminibus, quis opera Suetonii, quis Iuventii Martialis historiam quisve ad extremum Balbi ephemeridem fando adaequaverit?), aún era una fuente indispensable para conocer la figura del dictador. Por lo demás, es seguro que el propio Cornelio Balbo animara a Aulo Hircio a culminar la redacción de los Commentarii de bello Gallico, componiendo su libro VIII, que precisamente le dedica, ${ }^{12}$ y que su ejemplo cundiera entre algún otro partidario de César -y defensor de la causa de Octavio- que redactó las tres obras continuación de ésa, a saber el Bellum Hispaniense (obra tal vez del propio Hircio o de Gayo Opio), el Bellum Alexandrinum (también atribuido a Hircio) y el Bellum Africum (de nuevo atribuido a Hircio o a Opio). ${ }^{13}$ Bardon, incluso, llega a comparar el papel desempeñado en este sentido por Balbo en el entorno de César al de Mecenas en el entorno de Augusto. ${ }^{14}$

Su sobrino, Lucio Cornelio Balbo el Joven, nacido hacia el 80 a.C., participó también en las guerras civiles al lado de César, gozando plenamente de su confianza; en el 43 a.C., muerto el dictador, alcanzó la magistratura de cuestor, que desempeñó en la Hispania Ulterior, con lo que continuó incrementando enormemente la fortuna familiar; en el 21 a.C. se le confirió el proconsulado de África, donde derrotó a los garamantes, por lo que recibió en el 19 a.C. el honor del triunfo, ${ }^{15}$ siendo el primer extraitálico en alcanzarlo; tras él, nadie que no fuera de la familia imperial, volvería a lograr tan gran honor. Él fue quien mandó construir el teatro que lleva su nombre en la ciudad de Roma, dedicado el 13 a.C., con motivo del retorno de Augusto de la Galia.

11 Cfr. Julien 1886, 136-139.

12 Vid. Hirt., VIII pr. 1: Coactus adsiduis tuis vocibus, Balbe, cum cotidiana mea recusatio non difficultatis excusationem, sed inertiae videretur deprecationem habere, difficillimam rem suscepi.

13 De ser Hircio el autor de esas obras, debió de escribirlas entre marzo del 44, fecha de la muerte de César, y abril del 43, en que murió él mismo.

14 BARDON 1952, 282. Vid. también MüNZER 1900.

15 Vid. Plin., N.H. V 36: ultra eum deserta, Mathelgae oppidum Garamantum itemque Debris adfuso fonte a medio die ad mediam noctem aquis ferventibus totidemque horis ad medium diem rigentibus, clarissimumque Garama, caput Garamantum, omnia armis Romanis superata et a Cornelio Balbo triumphata, unius omnium curru externo et Quiritium iure donato; quippe Gadibus genito civitas Romana cum maiore Balbo patruo data est. Vid. GroAG 1900. 
También este Balbo hizo incursiones literarias, incluso más comprometidas intelectualmente que las de su tío, pues de él se dice que compuso un extenso tratado de cuestiones religiosas (quizás también gramaticales y de otros asuntos), ${ }^{16}$ titulado Exegetiká, fruto probablemente de sus conocimientos como pontifex y al que, sin duda, se refieren Macrobio (III 6.16: et Cornelius Balbus 'E $\xi \eta \gamma \eta \tau \iota \tilde{\omega} v$ libro octavo decimo ait apud aram Maximam observatum ne lectisternium fiat) y Servio (ad Aen. IV 127: Cornelius Balbus Hymenaeum ait, Magnetis filium, musicae artis peritum, pulchritudine muliebri, dum nuptias Liberi patris et Althaeae religiosis cantibus celebrat, exspirasse: propter quod ei talis honor in nuptiis adtributus est, ut celebratio nominis eius nuptiarum iungendarum perpetuum omen esset), $\mathrm{y}$ una tragedia pretexta cuyo nombre podría haber sido Iter ${ }^{17}$ y cuyo asunto, de acuerdo con Cicerón (ad Fam. X 32.3: Illa vero iam ne Caesaris quidem exemplo, quod ludis praetextam de suo itinere ad L. Lentulum pro consule sollicitandum posuit, et quidem, cum ageretur, flevit memoria rerum gestarum commotus) habría sido el fracaso de las negociaciones llevadas por él mismo ante Léntulo, en el marco de las contiendas civiles entre cesarianos y pompeyanos; esta tragedia, según el testimonio de Cicerón, habría sido representada a sus expensas en su Cádiz natal y le habría conmovido hasta hacerle llorar. ${ }^{18}$

Lucio Anneo Séneca, el Viejo, ${ }^{19}$ perteneciente al orden ecuestre, estudió en la escuela del grammaticus, en su Córdoba natal, ${ }^{20}$ pero ya a los quince años, en el 36 a.C., se trasladó a la capital con su buen amigo y compañero Porcio Latrón, al que ya se ha dicho, para seguir su formación bajo el rétor Marulo, de quien se supone también un origen hispano. Y, tras una estancia en Hispania para formar una familia $\mathrm{y}$, sin duda, para afianzar su patrimonio -rural, muy probablemente-, entre el 8 a.C. y el 5 d.C., regresa a Roma para dar en ella formación a sus tres hijos varones (Anneo Mela, Anneo Séneca y Anneo Novato); en este segundo viaje, le acompaña el hijo de otro notable cordobés, Clodio Turrino, de quien sabemos era un orador afamado. ${ }^{21}$ Si regresó o no a su Córdoba en los últimos años de su vida, es algo que no interesa

16 Varrón, L.L. 7.39 (nam et in Cornelii comentario erat ab Libycis Lucas, et in Virgilii ab Lucanis Lucas), cita un comentario de un tal Cornelio, que Bardon (1952, 198 n. 2) supone sea una obra titulada Expexégétiques $[$ sic], del propio Cornelio Balbo.

17 BARDon 1956, 177, sitúa entre los Flavios y los Severos a un Balbo, autor de un tratado, conservado incompleto, de geometría.

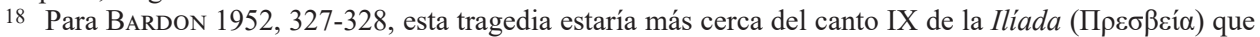
del Iter luciliano. Para esa praetexta, representada en el 43 a.C. en Gades, vid. Vell. Pat., II 51.3 (ed. de Hellegouarc'h), n. 5, p. 202.

19 Vid. ANDRÉ 1999.

20 Según su testimonio, la frecuentaban no menos de doscientos alumnos. Vid. Sen., Contr. I praef. 2: nam et duo milia nominum recitata quo erant ordine dicta reddebam, et ab his qui ad audiendum praeceptorem mecum convenerant singulos versus a singulis datos, cum plures quam ducenti efficerentur, ab ultimo incipiens usque ad primum recitabam. Vid. ANDRÉ 1999, 174: "La plus ancienne des provinces d'Occident [sc. Hispania] -après la Sicile- illustre un phénomène d'assimilation politique et culturelle dominé par l'urbanisation et la scolarisation des élites".

21 Vid. Sen., Contr. 10 praef. 14: Solebat declamare studiose et Turrinus Clodius, cuius filius fraterno vobis amore coniunctus est, adulescens summae eloquentiae futurus nisi mallet exercere quantum habet quam consequi quantum potest. 
ahora; ${ }^{22}$ en cualquier caso, sin duda pudo viajar a su ciudad de origen en numerosas ocasiones, pues no se tardaba más de 22 días en recorrer el trayecto entre Roma y la Bética, e incluso se podía hacer en menos si se consideraba preciso.

El testimonio de Séneca el rétor es aún más revelador de las profundas relaciones entre los provinciales y la capital: en sus Controversiae y en sus Suasoriae, cuyo título es en realidad Oratorum et Rhetorum Sententiae, Divisiones et Colores, ${ }^{23} \mathrm{da}$ cuenta de los numerosos declamadores -rétores o no- que llegó a conocer y tratar a lo largo de su dilatada vida (ca. 60/55 a.C.-ca. 40 d.C.; la obra parece escrita al final de la misma, hacia el 37 d.C.) ${ }^{24}$ en Roma o en Córdoba (o en cualquier otro lugar), muchos de ellos precisamente provinciales y, aún más, hispanos. ${ }^{25}$ No es este el momento para extenderme en los contenidos y en el significado de este gran corpus documental sobre la educación, la declamación y sus protagonistas en la Roma que él conoció; tampoco para explicar los no pocos problemas que plantea. ${ }^{26}$ Esta obra de Séneca el rétor no nos ha llegado completa, pero podemos hacernos una idea más que sobrada sobre su contenido, en especial sobre el contenido de la primera parte, las Controversiae, que constaban de diez libros, cada uno de ellos con un prefacio y un número variable de controversias, entre seis y nueve, de los que nos han llegado completos los libros I, II, VII, IX y X, mientras que los demás los conocemos por extractos. De las Suasoriae solo nos ha llegado el primer libro, con siete suasorias, de los dos de que debía de constar esa parte.

Una controversia es un ejercicio destinado a la formación judicial; su estructura es, por tanto, compleja y exige no solo un alto dominio de la técnica oratoria sino también una sólida formación intelectual en general y jurídica en particular, por más que los temas que se suelen tratar, propios del derecho civil y privado, en ocasiones parezcan, a pesar de sus intrincados argumentos, banales. En realidad, la variedad de temas es muy limitada, por lo que las repeticiones son constantes, lo que no impide que en ocasiones el tratamiento de estas cuestiones, ficticias o no, raye lo novelesco: hijos desheredados por razones absurdas, chicas violadas, asesinatos, adulterios, maquinaciones dolosas, etc.; naturalmente, todo ello provoca que los personajes sean estereotipos y más propios del teatro o de la novela que de la vida cotidiana. Mas todo ello resulta suficiente y útil para organizar un buen discurso, sostenido por razonadas y brillantes argumentaciones, y ese es el fin de estos ejercicios.

Por su parte, las suasorias son ejercicios mucho más simples, pues con ellos se espera tan solo que el orador sepa organizar un discurso apropiado para aconsejar a algún personaje relevante, histórico o legendario, lo que debería haber hecho en determinadas circunstancias y por qué. Obviamente, el alumno debe construir su dis-

22 Vid. GRIFFIN 1972, 8.

23 Abunda la bibliografía reciente dedicada a Séneca el rétor y a las declamaciones en Roma; además de la citada en otros lugares de este estudio, deben consultarse SuSSMAN 1978, FAIRWEATHER 1981, DUPONT 1997, Liell 1997, Lentano 1999, Gunderson 2003, Citti 2005, Berti 2007, Migliario 2007, 2008. Es un clásico la monografía de BONNER 1949.

24 Vid. GRIFFIN 1972, 11.

25 Vid. BornecQue 1902, André 1999, 171. Para la prosopografía en la obra de Séneca el rétor es imprescindible consultar EchaVARren 2007 y MASTROROSA 2008.

26 Vid. Adiego Lajara et al. 2005, 2005a. 
curso acopiando todo tipo de argumentos y ofreciendo todo tipo de datos, por lo que se exigía un alto grado de conocimientos de materias muy diversas.

Séneca organiza estos materiales de forma muy original, pues los discursos no se presentan completos, sino que se presenta la cuestión general seguida de la ley o leyes que le afectan y a continuación se ofrecen las opiniones o las soluciones ofrecidas por distintos declamadores a la cuestión planteada de acuerdo con una estructura tripartita (sententiae o frases u opiniones brillantes dichas al respecto, divisio o estructura del debate propuesto, y quaestiones o puntos de discusión sobre el tema y respuestas dadas). La finalidad de la obra de Séneca es esencialmente didáctica pues con ella pretende enseñar a sus hijos y a los estudiantes de las escuelas de retórica los métodos y las técnicas de organización y desarrollo de los discursos, mas los temas tratados aquí y las "citas" recogidas no pertenecen exclusivamente a un ambiente escolar sino que pueden proceder también de otros ámbitos profesionales.

Las declamaciones estaban de moda y en ellas se jugaban mucho de su prestigio y de su éxito los aspirantes a cargos públicos; la respuesta dada por Casio Severo al propio Séneca a propósito de su desapego por las declamaciones es bien ilustrativa de la situación. ${ }^{27}$ La formación intelectual que era preciso adquirir para lograr ser un orador aceptable era inmensa, las experiencias y conocimientos que se llegaban a adquirir ocupando cargos públicos, muy relevantes y, por tanto, la inclinación por poner todo ese bagaje al servicio de la consecución de una fama perdurable a través de la creación literaria, irreprimible. No pocos nobles, en efecto, probaron suerte con las letras, tratando de prestar un nuevo servicio al Estado mediante la redacción de una obra útil, habitualmente de carácter histórico, didáctico o, al menos, de docto entretenimiento. Y ése fue también el caso de los hispanos en la Roma de Augusto.

Es cierto que no todos los literatos y oradores de que tenemos noticia abandonaron sus lugares de origen; tal parece haber sido el caso de Clodio Turrino -cuyo hijo acompañó a los de Séneca en el segundo viaje a la Urbe- ${ }^{28}$ de Acilio Lucano -abuelo materno del poeta autor de la Farsalia - o de Gavio Silón, el orador que gustaba de escuchar Augusto durante su estancia en Tarragona en el 26-25 a.C. ${ }^{29}$ Sin duda, la existencia de un incipiente sistema educativo provincial, que contaba ya con escuelas de gramáticos -el primer peldaño escolar- destinadas a la formación romana de los hijos de los dirigentes locales, propiciaba que algunos hombres de letras pudieran ganarse su sustento sin necesidad de abandonar su patria chica; ${ }^{30}$ pero el anonimato de la mayoría de ellos o la nula pervivencia de las creaciones de aquellos cuyos nombres superaron la espesa capa del tiempo, evidencian que no era muy larga la carrera que se podía hacer ni siquiera en las capitales provinciales. Casos como el de Marsella,

27 Vid. Sen., Contr. III pr. 12: cum in foro dico, aliquid ago; cum declamo, id quod bellissime Censorinus aiebat de his qui honores in municipiis ambitiose peterent: uideor mihi in somniis laborare.

28 Quizás este Clodio Turrino sea el mismo que el gaditano Turriano Grácil, usado por Plinio en su Naturalis Historia como fuente en algunos lugares, que, además, se identifica con el praefectus Aegypti del año 4 a.C. y con el primer prefecto de la annona, que desempeñó el cargo entre el 14 y el 48 d.C., incluso a disgusto de Calígula. Vid. Demougin 1992, 372-373, nº 451.

29 Vid. Sen., Contr. 10 praef. 14: Bene declamavit Gavius Silo, cui Caesar Augustus, cum frequenter causas agentem in Tarraconensi colonia adisset, plenum testimonium reddidit.

30 Vid. MARrou 1950, ANDrÉ 1999, 175. 
a donde los patricios romanos llegaban a enviar a sus hijos para proporcionarles formación helénica de alto nivel, prefiriéndola en ocasiones incluso a Atenas, no son conocidos para el caso de Hispania, a pesar de la rápida latinización de buena parte de su territorio. ${ }^{31}$

Séneca el rétor, en efecto, da noticia de un enorme número de declamadores a los que tuvo ocasión de escuchar y con los que en muchas ocasiones pudo compartir o confrontar ideas, métodos y enseñanzas. Él es la principal fuente para la oratoria romana de la época postciceroniana. A través de sus Controversiae y de sus Suasoriae descubrimos que entre esos muchos declamadores abundan los de origen hispano, de modo que se ha llegado a sostener la existencia de un clan hispano -un lobby hispano- dependiente de los Anneos. De acuerdo con Echavarren, ahí están nada menos que unos 24 personajes para los que se puede atribuir un origen hispano (por más que no siempre Séneca lo indique), constituyendo de este modo el segundo grupo étnico en importancia de los mencionados por el rétor, ${ }^{32}$ tan solo por detrás de los de origen griego, que serían 44: ${ }^{33}$ Abronio Silón, padre, Clodio Sabino, Clodio Turrino, padre, ${ }^{34}$ Clodio Turrino, Cornelio Hispano, Fabio, L. Junio Galión, Marcio Marcelo, Mentón, Moderato, Papirio Fabiano, Paterno, Pompeyo Silón, (Servilio) Tusco, Fulvio Esparso, Gavio Sabino, Gavio Silón, Licinio Nepote, Manilio Nepote, Alfio Flavo, Marulo, ${ }^{35}$ M. Porcio Latrón, ${ }^{36}$ Estertinio Máximo y Séneca Grandión. A todos ellos tal vez convenga añadir los nombres de Estatorio Víctor ${ }^{37}$ y el del poeta cordobés Sestilio Ena. ${ }^{38}$ Es probable que algunos de ellos no hayan sido tratados personalmente por Séneca y que hayan llegado a la Urbe bastante antes de que lo hiciera el rétor cordobés, constituyendo de este modo una avanzadilla al aluvión de provinciales hispanos que llegarían en décadas posteriores, durante el principado de Augusto, primero, y de Tiberio, después; ese podría ser el caso de Catius Crispus, Saenianus, Statorius Victor, Seneca Grandio y, tal vez, de Fabius, Moderatus y Paternus. ${ }^{39}$ Mas casi todos ellos parecen enmarcados en un mismo ambiente romano, no provincial. Sin duda, esas relaciones, que a través de la obra senecana son tan solo de carácter literario y retórico, no se limitaban a estos aspectos, pues el conocimiento de la retórica y la práctica de la declamación no constituían necesariamente un fin en sí mismos, sino que tendían a lograr otros objetivos más ambiciosos, bien a través del desempeño de actividades públicas políticas o económicas, en el estado, en las provincias o en los municipios, bien a través de la práctica profesional de la enseñanza en calidad de rétores.

A los hombres de letras oriundos de Hispania mencionados por Séneca hay que añadir en estos primeros decenios del s. I d.C. el nombre de algún otro hispano nota-

31 Cfr. García y Bellido 1972.

32 ECHAVARREN 2007, 355.

33 Echavarren 2007, 330-347; Mastrorosa 2008, 70.

34 Vid. además de Echavarren s. u., Castillo 2006, 201 y supra n. 28.

35 Vid. además de Echavarren s. u., Castillo 2006, 202.

36 Vid. además de Bornecque 1902 y EChaVArRen, ANDré 1999, 175-177.

37 Castillo 2006, 202.

38 Castillo 2006, 202.

39 ECHAVARREN 2007, 356. 
ble como Cornelio Valeriano, mencionado por Plinio el Viejo entre sus fuentes.

Quizás también era originario de Hispania -aunque Alejandría le disputa ese mismo honor- el gramático, comentarista de la Eneida y prolífico escritor de fábulas mitológicas, temas astronómicos, agrícolas y otros, C. Julio Higino (64 a.C.-17 d.C.) $)^{40}$ que al igual que los anteriores ejerció su actividad en tiempos de Augusto, de quien era liberto, llegando a ser bibliotecario de la biblioteca palatina y granjeándose la amistad del poeta Ovidio; tal vez el poeta de Sulmona le dedicó el libro III de sus Tristia, si es que es él el que se esconde bajo la expresión cultor et antistes doctorum sancte virorum ( $T r$. III 14.1), como quieren algunos. Habría llegado a Roma aún muy joven como propiedad de Julio César, habría seguido las enseñanzas de Alejandro Polyhístor y sin duda sus extraordinarias dotes intelectuales le debieron propiciar el favor de su dueño - quizás ya el propio Augusto-, que le concedió la libertad y el nombre. Suetonio es el primero que, en lo que nosotros sabemos, le atribuye origen hispano, de acuerdo con lo dicho en Gram. 20 y no sin dudas, pues se hace eco también de su presunto origen alejandrino. ${ }^{41}$ Su peripecia vital quiso, no obstante, que conociera el infortunio y consecuentemente la pobreza, de la que, al parecer, le sacó el consular Claudio Licinio. Puede ser que el Higinio de Suetonio sea el mismo mencionado por Columela ${ }^{42}$ Asconio Pediano, ${ }^{43}$ Plinio, ${ }^{44}$ Aulo Gelio,${ }^{45}$ Servio $^{46}$ y Macrobio,${ }^{47}$ con cuyas citas y referencias se puede dibujar la biografía literaria de este prolífico escritor, si es que es uno solo, como quieren Boriaud y Del Hoyo-García Ruiz, por ejemplo. A tenor de esas informaciones, pues, su obra incluiría un De familiis Troianis (Seru., in Aen. II 15; V 389), en la línea del tratado homónimo de Varrón y que convendría no poco al ambiente augusteo, un De agri cultura (o De re rustica; Col., III 11.8; XI 2.83; 3.62; Plin., N.H. XIII 24; XVI 43; XVIII 26, etc.) y un De apibus (Col., IX 2.1-

40 Vid. Duret 1983, 1539-1543, Boriaud 1997, Del Hoyo-García Ruiz 2009, donde se ofrecen diversos y recientes estado de la cuestión; aunque no siempre son coincidentes - como no podía ser menos- las respuestas a los numerosos enigmas que plantea este (o estos) Higino(s), parece imponerse cierto consenso a propósito de atribuir idéntica paternidad a las Fabulae y a la Astronomica y en situar ambas obras en el ambiente cultural de Augusto.

41 Suet., Gram. 20: C. Iulius Hyginus Augusti libertus, natione Hispanus, - nonnulli Alexandrinum putant et a Caesare puerum Romam adductum Alexandria capta - studiose et audiit et imitatus est Cornelium Alexandrum grammaticum Graecum quem propter antiquitatis notitiam Polyhistorem multi, quidam Historiam vocabant. praefuit Palatinae bibliothecae nec eo secius plurimos docuit fuitque familiarissimus Ovidio poetae et Clodio Licino consulari historico qui eum admodum pauperem decessisse tradit et liberalitate sua quoad vixerit sustentatum. huius libertus fuit Iulius Modestus in studiis atque doctrina vestigia patroni secutus. Nuestro Juan Luis Vives, que editó su Poeticon Astronomicon (París, 1514), siendo esta su primera publicación (París, 1514), lo apreciaba sobremanera (¿quizás por considerarlo paisano?), según se desprende de las numerosas referencias que hace de él a propósito de temas muy variados. Cfr. http://bivaldi.gva.es/estaticos/contenido. cmd?pagina $=$ estaticos/vives/vives_inicio.

42 Vid. Col., I 1.13; III 11.8; IX 2.1-2; 11.5; 13.3; 13.6; 13.8; 14.1; 14.18; XI 2.83; 3.62.

43 Vid. Ped., Asc. ad Cic. in Pis. 12: Varronem autem tradere M. Valerio, quia Sabinos vicerat, aedes in Palatio tributas, Iulius Hyginus dicit in libro priore de viris claris, $<e t>$ P. Valerio Volesi filio Publicolae aedium pub<licelo>cum sub Veliis, ubi nunc aedis Victoriae est, populum ex lege quam ipse tulerat concessisse.

44 Vid. Plin., N.H. passim.

45 Vid. Gel., Pr. 1.21; 5.8; 7.6; 10.16; 16.6; I 14.1; 21, pr.; 21.2-4; V 8, pr.; 8.1; 8.3; VI 1.2; VII 6, pr.; 6.2; 6.5; X 16, pr.; 16.1; 18.7; XVI 6, pr.; 6.14-15.

46 Vid. Serv., in Aen. I 277; 530; II 15; III 553; V 389; VII 47; 412; 678; VIII 597; 600; 638; XII 120.

47 Vid. Macr., I 7.19; V 18.16; III 4.13; III 8.4; VI 9.7. 
$2 ; 13.8)$, ambos también en una línea muy virgiliana al igual que un De dis penatibus (Macr., III 4), un De proprietatibus deorum (Macr., III 8), unos Exempla (Gel., X 18.7), que quizás sea la misma obra que otra cuyo título sería De uita rebusque illustrorum uirorum (Gel. I 14) o que la titulada De uiris claris (Asc., ad Cic. in Pis. 12), un De situ urbium italicarum (Seru., in Aen. I 277; 530; III 553; VIII 600; VIII 638; Macrob., I 7.19), ninguna de ellas conservada, además de las ya mencionadas fábulas mitológicas y los Astronomica, que sí han llegado a nosotros. En todas ellas, en las conservadas y en las no conservadas, parece haber unas líneas comunes de notable base mitológica, mas nada hay que pueda justificar, desde la producción conservada, su adscripción a Hispania. ${ }^{48}$

A partir de este momento, la literatura latina será durante mucho tiempo una literatura hecha en buena medida por hispanos y en ella se han buscado rasgos específicos de ese origen. ${ }^{49}$ Pomponio Mela, el geógrafo nacido en Tingentera-Algeciras(principios del I d.C. y muerto en el 45 d.C.), Columela, el tratadista de agricultura, también de Gades (4 d.C. - ca. 70 d.C.) y también ligado al círculo de los Anneos, o Lucio Cornelio Boco $^{50}$ anuncian, en tiempos de Claudio, la eclosión definitiva de la literatura hispanolatina, particularmente intensa en el período neroniano y bajo la dinastía Flavia.

\section{Bibliografía}

AdAMS, J.N. (2007): The regional diversification of latin 200 BC-AD 600, Cambridge.

Adiego Lajara, I.J.-Artigas Álvarez, E.-Riquer Permanyer, A. DE (2005): Séneca el Viejo, Controversias. Libros I-V, introducción, traducción y notas de ---, Madrid.

(2005a): Séneca el Viejo, Controversias. Libros VI-X. Suasorias, introducción, traducción y notas de ---, Madrid.

Almagro Gorbea, M. (2010): “Lucio Cornelio Boco: Turdetano de Salacia y autor de la Edad del Plata de la Literatura Latina", Estudos Arqueológicos de Oeiras 18, 287-332.

Alvar Ezquerra, A.

(1983): "Para una sociolingüística del latín", [en] Philologica Hispaniensia in honorem M. Alvar. I. Dialectología, Madrid, 57-68.

(1998): "El latín y la dialectología", La Torre. Revista de la Universidad de Puerto Rico III, 111-149.

(1998a): "Escritores latinos de Hispania", [en] Hispania. El legado de Roma (en el año

48 Tampoco queda demostrada de modo indubitable esta filiación en LASSO DE LA VEGA 1974.

49 Vid. Rostagni 1983, 404: "L'origine spagnuola [sc. de Séneca el rétor] poi lo disponeva evidentemente a certe forme stilistiche che sono legate alla retorica. Egli fu il primo di tutta una importante schiera di autori che la Spagna diede nel I secolo alla letteratura latina; i quali contribuirono tutti più o meno a versare in questa letteratura una special vena di stile turgido e barocco, ricco di colori, di fregi e di "acutezze": poichè già Cicerone negli Spagnuoli indicava alcunchè di enfatico e stravagante, pingue quoddam et peregrinum (nella Pro Arch. 10.26: un passo ch'è ricordato da Seneca stesso, Suas. 6.27). Vid. también GrIfFIN 1972, pp. 14-15. Alvar 1998, 1998a, ANDré 1999, 171 y ss. Para los intelectuales hispanos cuyos nombres nos han llegado a través de la epigrafía, vid. CASTiLlo 1990 y CABAllos (passim).

50 Vid. para L. Cornelio Boco, Almagro Gorbea 2010, Alvar Ezquerra 2011. 
de Trajano) [Catálogo de la Exposición celebrada en La Lonja, Zaragoza, septiembre noviembre 1998], Ministerio de Educación y Cultura, 281-288 (= Hispania. El legado de Roma (en el año de Trajano) [Catálogo de la Exposición celebrada en el Museo Nacional de Arte Romano, Mérida, febrero - abril, 1999], Ministerio de Educación y Cultura, 1999, 317-323).

(2011): "Más notas de asedio a Lucio Cornelio Boccho", [en] J. L. Cardoso - M. AlmagroGorbea (eds.), Lucius Cornelius Bocchus. Escritor Lusitano da Idade de Prata da Literatura Latina. (Colóquio Internacional de Tróia, 6-8 de Outubro de 2010), Lisboa- Madrid, 259-274.

ANDrÉ, J.-M. (1999): “Les Sénèques et l’Espagne”, REL 77, 170-183.

BARDON, H.

(1952): La littérature latine inconnue I. L'époque républicaine. Paris.

(1956) La littérature latine inconnue II. L'époque impériale, Paris.

Berti, E. (2007): Scholasticorum Studia. Seneca il Vecchio e la cultura retorica e letteraria della prima età imperiale, Pisa.

Bonner, S.F. (1949): Roman Declamation in the late Republic and early Empire, Liverpool.

Boriaud, J.-Y. (1997): Hygin, Fables, ed. y trad. de ---, Paris.

Bornecque, H. (1902): Les déclamations et les déclamateurs d'après Sénèque le père, Lille (= reimpr. Hildesheim, Olms, 1967).

Boscs-Plateaux, F. des (1994): "L. Cornelius Balbus de Gadès: la carrière méconnue d'un espagnol à l'époque des Guerres civiles (1 ${ }^{\mathrm{er}}$ siècle av. J.-C.)", Mélanges de la Casa de Velázquez 30.1, 7-35.

Caballos Rufino, A.

(1986): "La romanización de las ciudades de la Bética y el surgimiento de senadores provinciales", Revista de Estudios Andaluces 6, 13-26.

(1989): "Los senadores de origen hispano durante la República romana", [en] Estudios sobre Urso Colonia Iulia Genetiva, Sevilla, 233-279.

(1990): Los senadores hispanorromanos y la romanización de Hispania (siglos I-III). I: Prosopografía, Écija (Sevilla).

(1998): "Cities as the basis for supra-provincial promotion: the equites of Baetica", [en] S. Keay (ed.), The Archaeology of early Roman Baetica, Portsmouth, Rhode Island, (=Journal of Roman Archaeology, Suplemment no 29) 123-146.

(1998): "Los equites y la dinámica municipal de la Lusitania, I: Catálogo Prosopográfico", [en] El proceso de municipalización en la Hispania romana. Contribuciones para su estudio, Valladolid, 205-233.

(1999): "Preliminares sobre los caballeros romanos originarios de las provincias hispanas. Siglos I-III d.C.)", [en] J. F. Rodríguez Neila - F. J. Navarro Santana (eds.), Élites y promoción social en la Hispania Romana, Pamplona, 103-144.

(1999a): "Los caballeros romanos originarios de las provincias de Hispania. Un avance", [en] S. Demougin - H. Devijver - M.-Th. Raepsaet-Charlier (eds.), L'ordre équestre. Histoire d'une aristocratie (II' siècle av. J.-C.- III' siècle ap. J.-C., Roma, 463-512.

(2001): "Der Aufstieg Lokaler Eliten Spaniens in die Reichselite", [en] L. De Blois (ed.), Administration, Prosopography and Appointment Policies in the Roman Empire. Proceedings of the First Workshop of the International Network 'Impact of Empire' (Roman Empire, 27 B.C. - A.D. 406), Amsterdam, 255-271. 
(2001): "Las Elites y el Poder", [en] M. Navarro Caballero - S. Demougin (ed.), Élites Hispaniques, Bordeaux, 187-189.

(2006): "Implantación territorial, desarrollo y promoción de las elites de la Betica", [en] A. Caballos Rufino y S. Demougin (eds.), Migrare: La formation des élites dans l'Hispanie romaine, Bordeaux, 241-271.

(2009): "La extracción de hispanos para formar parte de la aristocracia imperial: senadores y caballeros", [en] J. Andreu Pintado - J. Cabrero Piquero - I. Rodà de Llanza (eds.), Hispaniae. Las provincias hispanas en el mundo romano, Tarragona, 265-281.

Castillo García, C.

(1965): Prosopographia Baetica, 2 vols, Pamplona.

(1990): "Personas y familias notables en la Bética romana", [en] De la Grecia arcaica a la Roma imperial, Madrid, 135-147.

(1984): "Los senadores de la Bética: onomástica y parentesco", Gerión 2, 239-254 (=Vestigia Antiquitatis, Pamplona, 1997, 443-455).

(2006): “Missing persons?: algunos literatos de origen bético relacionados con los Sénecas y con Marcial", [en] C. Santini, L. Zurli, L. Cardinali (eds.), "Concentus ex dissonis": scritti in onore di Aldo Setaioli vol. I, Napoli, 201-205.

CitTi, F. (2005): "Elementi biografici nelle Prefazioni di Seneca il Vecchio", Hagiographica $12,171-222$.

Del Hoyo, J. - García Ruiz, J. M. (2009): Higino, Fábulas, introd. y trad. de ---, notas e índices de J. Del Hoyo, Madrid.

Demougin, S. (1992): Prosopographie des chevaliers romains Julio-Claudiens (43 av. J.C.-70 ap. J.C.), Roma.

Dupont, F. (1997): "Recitatio and the reorganization of the space of public Discourse", [en] T. Habinek y A. Schiesaro (eds.), The Roman Cultural Revolution, Cambridge, 44-59.

Duret, L. (1983): "Dans l'ombre des plus grands: I. Poètes et prosateurs mal connus de l'époque augustéenne", [en] $A N R W$, II, 30, 3, Berlin-New York, 1447-1560.

Echavarren, A. (2007): Nombres y personas en Séneca el Viejo, Pamplona.

FAIRweATHER, J. (1981): Seneca the Elder, Cambridge.

García y Bellido, A. (1972): “Die Latinisierung Hispaniens”, [en] $A N R W, \mathrm{I}, 1$, Berlin-New York, 462-500.

GrIFFIN, M. (1972): “The Elder Seneca and Spain”, JRS 62, 1-19.

Groag, E. (1900): s. v. “L. Cornelius Balbus der Jüngere”, [en] RE 4, 1268-1271 (nº 70).

Gualandri, I. (1989), "Per una geografia della letteratura latina", [en] G. Cavallo-P. FedeliA. Giardina (dirs.), Lo spazio letterario nella antica Roma, II. La circolazione del testo, Roma, 476-484.

Gunderson, E. (2003): Declamation, Paternity, and Roman Identity. Authority and Rhetorical Self, Cambridge.

Julien, E. (1886): De P. Cornelio Balbo maiore.

LASSO DE LA Vega, J. (1974): “Cayo Julio Hyginio, primer bibliotecario español”, Revista de Archivos, Bibliotecas y Museos 77.2, 435-447.

LENTANO, M. (1999): "La declamazione latina. Rassegna di studi e stato delle questioni (19801998)", BStudLat 29, 571-621. 
LIELL, S. (1997): "Die politischen Ambitionen des älteren Seneca. Überlegungen zu Sen. contr. 2, praef. 4", [en] B. Czapla - T. Lehmann - S. Liell (eds.), Vir bonus dicendi peritus. Festschrift für Alfons Weische zum 65. Geburtstag, 261-270.

Marrou, H. I. (1950): Histoire de l'éducation dans l'Antiquité, Paris (trad. esp. FCE).

Mastrorosa, I.G. (2008): "La prosopografia della cultura retorica fra Augusto e Tiberio: in margine a uno studio recente su Seneca il Vecchio", BStudLat 38, 62-74.

MigLiario, E.

(2007): Retorica e storia. Una lettura delle Suasoriae di Seneca Padre, Bari.

(2008): "Cultura politica e scuole di retorica a Roma in età augustea", [en] F. Gasti - E. Romano (eds.), Retorica ed educazione delle élites nell'antica Roma: atti della VI giornata ghisleriana di filologia classica (Pavia, 4-5 aprile 2006), Como, 77-93.

Münzer, F. (1900): s. v. “L. Cornelius Balbus”, [en] RE 4, 1260-1268 (nº 69).

Padilla Monge, A. (2006): "La integración de las oligarquías indígenas en las elites coloniales del sur de Hispania", [en] A. Caballos Rufino - S. Demougin (eds.), Migrare: La formation des élites dans l'Hispanie romaine, Bordeaux, 205-240.

Rodríguez Neila, J. F. (1996): Confidentes de César. Los Balbos de Cádiz, Madrid.

Rostagni, A. (1983): Storia della letteratura latina. II. L'impero. Parte prima: da Augusto a Nerone, (tercera edición revisada y ampliada por I. Lana, Torino, 1964).

RuBIO, L.

(1949): “Los Balbos y el Imperio Romano”, Anales de Historia Antigua y Medieval (Buenos Aires), 67-119.

(1950): "Los Balbos y el Imperio Romano", Anales de Historia Antigua y Medieval (Buenos Aires), 142-199.

(1954): M. Tulio Cicerón, Defensa de L. C. Balbo, introd., ed. y com. por ---, Barcelona.

Saquete Chamizo, J. C. (2006): "La integración de las elites hispanas en Roma. El caso de la religión pública y los senadores béticos" [en] A. Caballos Rufino - S. Demougin (eds.), Migrare: La formation des élites dans l'Hispanie romaine, Bordeaux, 301-337.

Sussman, L.A. (1978): The Elder Seneca, Leiden.

WeInrib, E. J. (1990): The Spaniards in Rome: From Marius to Domitian, London. 